\title{
Propuesta de muestreo para Neohydatothrips signifer (Thysanoptera:Thripidae) en el cultivo de maracuyá
}

\author{
Oscar Santos Amaya ${ }^{(1)}$, Edgar Herney Varón Devia( ${ }^{(2)}$ y Johana Andrea Floriano ${ }^{(2)}$
}

(1)Universidade Federal de Viçosa, Laboratório de Interação Inseto-Planta, CEP 36570-000 Viçosa, Minas Gerais, Brasil. E-mail: santosamaya@gmail.com ${ }^{(2)}$ Corporación Colombiana de Investigación Agropecuaria, Km 12 Vía Espinal-Ibagué, CEP 730001, Ibagué, Colombia. E-mail: evaron@corpoica.org.co, andreafloriano80@yahoo.es

Resumen - El objetivo de este trabajo fue desarrollar una propuesta de muestreo de Neohydatothrips signifer en el cultivo de maracuyá. Se correlacionó la infestación promedio del trips en el cultivo con las variables meteorológicas de humedad relativa, temperatura y precipitación, con uso del coeficiente de correlación de Pearson. Así mismo, se determinó el índice de agregación de la población en el cultivo, con la prueba chi cuadrado $\left(\mathrm{X}^{2}\right)$ para la distribución de Poisson, la prueba de la razón $\mathrm{V}^{2} / \mu$, y la distribución de la binomial negativa. La única variable climática que se correlacionó significativamente con la densidad promedio del trips fue la temperatura. Se presentó mayor incidencia y densidad poblacional del insecto en los terminales vegetativos en comparación con los botones florales. El patrón de disposición espacial que se presentó en todos los estados de desarrollo fenológico del cultivo fue de tipo agregado. Con el índice de agregación y el tamaño promedio de la población, se calculó que se deben muestrear 22 terminales nuevos por hectárea, sin importar su posición en la planta. El muestreo del trips en el cultivo de maracuyá debe hacerse sobre los terminales vegetativos de la planta, sin importar la posición del terminal en ella.

Términos para indexación: Passiflora edulis f. flavicarpa, distribución espacial, fluctuación poblacional, índice de agregación, trips.

\section{Proposed sampling for Neohydatothrips signifer (Thysanoptera:Thripidae) in passion fruit crop}

\begin{abstract}
The objective of this work was to develop a proposal for Neohydatothrips signifer sampling in the passion fruit crop. Average thrip infestation on the crop was correlated with the meteorological variables of relative humidity, temperature, and precipitation using Pearson correlation coefficient. Likewise, determinations were done for the aggregation rate of the growing population using chi square $\left(\mathrm{X}^{2}\right)$ for the Poisson distribution, the ratio test $\mathrm{V}^{2} / \mu$ and the negative binomial distribution. Temperature is the only climatic variable that significantly correlated with the mean density of the thrips. There were higher incidence and population density of the insects at the vegetative terminal buds, in comparison to the flower buds. The spatial distribution pattern for this insect, along all phenological stages of the plant, was aggregated. Using the aggregation index and the average population, a sample of 22 new vegetative terminal buds per hectare was determined as appropriate, regardless of its position within the plant. A thrip sampling method in the passion fruit crop should sample over terminal buds, without account their position on plants.
\end{abstract}

Index terms: Passiflora edulis f. flavicarpa, spatial distribution, population fluctuation, agreggation index, thrips.

\section{Introducción}

El maracuyá (Passiflora edulis Degener) se cultiva comercialmente en Colombia en 19 departamentos, de los cuales Huila, Valle del Cauca, Córdoba y Meta son los principales productores (Jaramillo Vásquez et al., 2009). Neohydatothrips signifer (Priesner, 1932) (Thysanoptera: Thripidae) es la principal plaga del cultivo de maracuyá en el departamento del Huila (Salamanca Bastidas et al., 2010). En esta región, se registró que este insecto puede causar una reducción de $311 \mathrm{~kg} \mathrm{ha}^{-1}$ de la fruta, cuando el nivel de infestación promedio de la población en el cultivo aumenta en una unidad (trips por terminal) (Santos A. et al., 2012). Este alto daño ha propiciado que los agricultores y asistentes técnicos en la zona tomen medidas aceleradas para su control, basadas fundamentalmente en el uso de productos químicos, algunos de los cuales son de categoría toxicológica I y II y de amplio espectro (Santos A. et al., 2012). El uso indiscriminado de ese tipo de 
insecticidas puede ocasionar problemas de manejo en el futuro, como resistencia a los insecticidas (Chen et al., 2011) y restricciones para la comercialización en los mercados internacionales (Arturo Guerrero, 2003).

Una de las formas de reducir el uso de aplicaciones de insecticidas y evitar las aplicaciones por calendario y sus posibles efectos colaterales dentro de un sistema de manejo es establecer y hacer uso del nivel de daño económico (NDE) (Bueno \& Cardona, 2003). Para hacer uso del NDE, es necesario estimar la densidad poblacional de la plaga en el cultivo, para lo cual se requieren métodos eficaces y precisos de muestreos en campo, sin incurrir en costos excesivos (González-Zamora \& Garcia-Marí, 2003; Kovanci et al., 2005). Desarrollar métodos de muestreos en insectos plagas es dificultoso ya que es necesario el conocimiento previo de algunos parámetros ecológicos de la población, como lo son la distribución espacial y la fluctuación poblacional, entre otros (Ramírez-Dávila et al., 2002).

En Colombia, el NDE para N. signifer fue establecido por Santos A. et al. (2012); sin embargo, no se conocen estudios sobre muestreos de este insecto en el cultivo, lo que ha impedido realizar un manejo eficiente y racionalizado de la plaga. Establecer un sistema de muestreo del trips del maracuyá se constituye como investigación de base para el control exitoso de este insecto. La generación de este conocimiento podría definir con mayor exactitud la peligrosidad del trips como plaga del maracuyá y orientaría a los agricultores en la toma de decisiones para el control del trips, dentro del contexto del manejo integrado de plagas (MIP) (Nault \& Shelton, 2010).

El objetivo del presente trabajo fue desarrollar una propuesta de muestreo para Neohydatothrips signifer en el cultivo de maracuyá con base en las orientaciones del MIP.

\section{Materiales y Métodos}

Los experimentos se desarrollaron en el Municipio de Suaza (Huila), vereda San Isidro, finca El Lago, a $01^{\circ} 57^{\prime} 48^{\prime \prime} \mathrm{N}, 075^{\circ} 47^{\prime} 21$ "W y $900 \mathrm{~m}$ de altitud, con temperatura y humedad relativa promedio de $24^{\circ} \mathrm{C}$ y $49 \%$ respectivamente.

Para determinar la fluctuación poblacional del trips, con respecto a los estados de desarrollo fenológico del cultivo y a las variables meteorológicas de temperatura, humedad relativa y precipitación, se establecieron seis parcelas de $20 \mathrm{~m}$ de largo por $10 \mathrm{~m}$ de ancho. En cada parcela, se sembraron 16 plantas de maracuyá, distribuidas en cuatro surcos, cada uno con cuatro plantas distanciadas entre sí cuatro metros; la distancia entre surcos fue de dos metros.

Los muestreos se efectuaron dos veces por semana, en ocho plantas tomadas aleatoriamente por parcela. El conteo se hizo sobre los terminales vegetativos de la planta, habiéndose discriminado el trips por su estado de desarrollo de ninfa o adulto. Para el muestreo, se utilizaron dos procedimientos: golpeo, que consistió en sacudir tres veces cada uno de los terminales de la planta sobre una cartulina de color blanco, en que todos los trips fueron contados; y "visual", a través de lo cual el terminal vegetativo fue cuidadosamente examinado in situ, para contar los trips observados (González-Zamora \& Garcia-Marí, 2003). Los muestreos se realizaron durante el segundo semestre de 2009 y el primero de 2010, desde el establecimiento del cultivo hasta las primeras cosechas. Se tomaron en campo datos diarios de humedad relativa, precipitación y temperatura. En el manejo fitosanitario del cultivo no se aplicaron insecticidas; las demás prácticas agronómicas se realizaron de acuerdo con las recomendaciones de los agricultores de la región (García Lozano et al., 2007).

Para el análisis de los datos, se realizaron correlaciones entre número de trips por semana $\mathrm{y}$ los datos meteorológicos de precipitación, humedad relativa y temperatura. Estas correlaciones se hicieron de dos formas, la primera correlacionando el número de trips por semana con los datos de las variables meteorológicas tomadas en la misma semana, y la segunda con los datos de las variables meteorológicas de cada una de las tres semanas anteriores (semanas rezagadas), para ver los efectos que tienen estas variables sobre la densidad poblacional del trips en muestreos posteriores. Así mismo, se realizaron correlaciones múltiples entre las variables meteorológicas con el número de trips por semana. Las correlaciones se realizaron con el coeficiente de Pearson y el procedimento CORR, disponible en el programa estadístico SAS. Para relacionar la fluctuación poblacional del trips con los estados de desarrollo fenológicos de la planta, se compararon gráficamente las densidades poblacionales registradas del insecto en cada uno de esos estados. Los estados de desarrollo fenológico del cultivo de maracuyá, que se registraron en campo, fueron: etapa vegetativa V1, fase de post-trasplante - desde el trasplante hasta que 
la planta alcanzó la altura del alambre en el cual ella se ramifica, aproximadamente $1,70 \mathrm{~m}$; etapa vegetativa V2, desarrollo vegetativo - ocurrió durante los 100 a 120 días después de la emergencia, en que la planta únicamente produjo estructuras vegetativas; etapa vegetativa V3, aparición de los primeros botones florales (a partir del quinto mes) - en que se observó una superposición entre la fase vegetativa y reproductiva; etapa reproductiva $\mathrm{R} 1$, que correspondió a la floración, en que más del $50 \%$ de las plantas presentaron flores en cartucho y abiertas; etapa reproductiva $\mathrm{R} 2$, denominada de formación del fruto - tuvo una duración aproximada de 50-60 días; etapa reproductiva R3 - corresponde a los procesos de llenado y maduración del fruto y presentó una duración de 20 a 25 días (Rivera et al., 2002).

Se determinó la incidencia del trips en los botones florales y terminales vegetativos, con el registro de la presencia o ausencia del insecto en estas estructuras vegetativas; para ello, se utilizaron las parcelas establecidas para el estudio de fluctuación poblacional; no se examinaron frutos debido a que no se encontró incidencia del insecto en estos órganos en campo. Para determinar diferencias estadísticas entre las dos estructuras en cuanto a la incidencia, se realizó una prueba de $t$, se tomaron las seis parcelas como réplicas y las dos estructuras (botones y terminales) como tratamientos. Se utilizó el procedimiento TTEST, disponible en el programa estadístico SAS. Estos análisis se realizaron para adultos y ninfas en conjunto y para cada uno por separado. Así mismo, se determinó qué estructura alberga el mayor número de trips; para ello, se registró la cantidad de individuos encontrados por estructura. El análisis de los datos se hizo siguiendo los procedimientos descritos para determinar la incidencia del trips por estructura.

Dado a que el cultivo de maracuyá no presenta los estratos bajo, medio y alto, debido a la arquitectura de la planta de tipo enredadera, se procedió a medir la incidencia y densidad del trips, por la división de la rama principal de la planta por tercios $(1,2$ y 3$)$. El primero correspondió a la parte de la rama próxima al tallo principal, el segundo a la parte media y el tercero a la parte final de la rama. En cada uno de los tercios se muestreó un terminal y un botón floral tomados aleatoriamente. El conteo de los individuos se realizó dos veces por semana, durante doce meses, con discriminación de ninfas y adultos.
La incidencia del trips por tercios se determinó por el registro de la presencia o ausencia del insecto por estructura (botón y terminal) en cada uno de los tercios. De esta forma, se obtuvieron los porcentajes de incidencia del insecto, por estructura, para cada estrato. Para determinar diferencias estadísticas entre los estratos en cuanto a incidencia, se realizó un análisis de varianza (Anova) y la prueba de Tukey, para determinar las diferencias de medias entre los tratamientos. Se tomaron las seis parcelas como réplicas y los tercios como tratamientos. Para el análisis de los datos, se utilizó el procedimiento GLM, disponible en el programa estadístico SAS. Estos análisis se realizaron para adultos y ninfas en conjunto y para cada uno por separado. Igualmente, se determinó en qué estrato es mayor la densidad poblacional del insecto; para ello, se registró la cantidad de individuos encontrados, por estructura, en cada uno de los tercios.

Para determinar el patrón de disposición de $N$. signifer en el cultivo, se tomaron 48 plantas distribuidas equidistantemente sobre el terreno, sin obviar los bordes; estas plantas fueron marcadas y utilizadas durante todo el estudio. Los muestreos se realizaron semanalmente durante diez meses, $y$ tres terminales por planta fueron revisados, sin discriminar los trips por su estado de desarrollo. El patrón de disposición agregada, al azar o uniforme, para el número de trips por terminal, por planta, se determinó a través de la distribución de Poisson, empleándose: la prueba de chi-cuadrado; la prueba de la razón [varianza/media $\left.\left(\mathrm{V}^{2} / \mu\right)\right]$, en que, las relaciones de varianza/media mayores que 1 indican un patrón de distribución agregada, las iguales a 1 indican la disposición al azar, y las menores que 1 indican disposición uniforme (Pérez \& Iannacone, 2009); y, por último, la distribución binomial negativa, en que el valor de K permite definir el índice de agregación (Michela et al., 2000), y cuanto menor es el valor de $\mathrm{K}$, mayor es la intensidad de agregación $\mathrm{y}$, si $\mathrm{K}$ es grande $(K>8)$, la distribución es al azar (Pérez \& Iannacone, 2009). Para la estimación de los parámetros de la binomial negativa y la distribución de Poisson, se utilizó el procedimiento Genmod, disponible en el programa estadístico SAS. Según Madadi et al. (2011), los trips muestran pautas de distribución agregativas, $\mathrm{y}$ son bastante raras las aleatorias y las uniformes. Bajo esta afirmación, la fórmula para determinar el número de muestras a tomar de una plaga está representada por el modelo matemático binomial negativo (Aranda 
González, 2004), cuya fórmula desarrollada por Rojas (1964) es: $n=(1 / \mu+1 / k) / \mathrm{CV}^{2}$ en que: $n$ es el numero de muestras; $\mathrm{k}$ es el índice de dispersión de la distribución binomial negativa; $\mu$ es la media de la población del insecto, estimada en los muestreos; y CV es el coeficiente de variación. Rojas (1964) estimó que, para este tipo de estudios, un buen $\mathrm{CV}$ es de 0,3 .

\section{Resultados y Discusión}

La temperatura fue la única variable meteorológica que se correlacionó positivamente con la población promedio semanal de trips adultos, en la misma semana de muestreo (Figura 1) y para una semana rezagada. El aumento de la temperatura tuvo una respuesta positiva en el incremento de la densidad poblacional del trips. En las otras variables, no se presentaron correlaciones significativas. En los análisis de correlación múltiple, entre las variables meteorológicas con la densidad poblacional promedio del trips, se encontró que las únicas variables que se relacionaron significativamente fueron la precipitación en la misma semana, y la temperatura con un rezago de una semana (Pearson, $\mathrm{p}<0,05)$. Esta relación indica que cuando en la semana de muestreo no se presentan precipitaciones, y esta es antecedida por una semana con temperaturas altas, es de esperarse un aumento en el número de trips. La tendencia registrada en este estudio coincide con otras investigaciones, en las que se relató que los periodos secos y de altas temperaturas favorecen el desarrollo poblacional de los trips, lo que aumenta su población (Ávila Quezada et al., 2002; Urías-López et al., 2007). En general, el efecto que ejerce la temperatura sobre los insectos, se debe a que cuando la temperatura ambiental baja, la temperatura corporal del insecto también lo hace, y sus procesos fisiológicos se reducen (Triplehorn \& Johnson, 2011). Por eso, este factor es el que ejerce un mayor efecto sobre su desarrollo (Marco, 2001). Lo anterior ya ha sido evidenciado para trips por Park et al. (2010), quienes registraron sobre Thrips palmi que el tiempo de desarrollo del insecto disminuye en todas las etapas de desarrollo con el aumento de las temperaturas.

En cuanto a la fluctuación poblacional del insecto con respecto a los estados de desarrollo fenológico del cultivo, se encontró que la menor población del trips se presentó en los estados de desarrollo vegetativos V1 y V2, evidenciándose un aumento en el estado V3 en

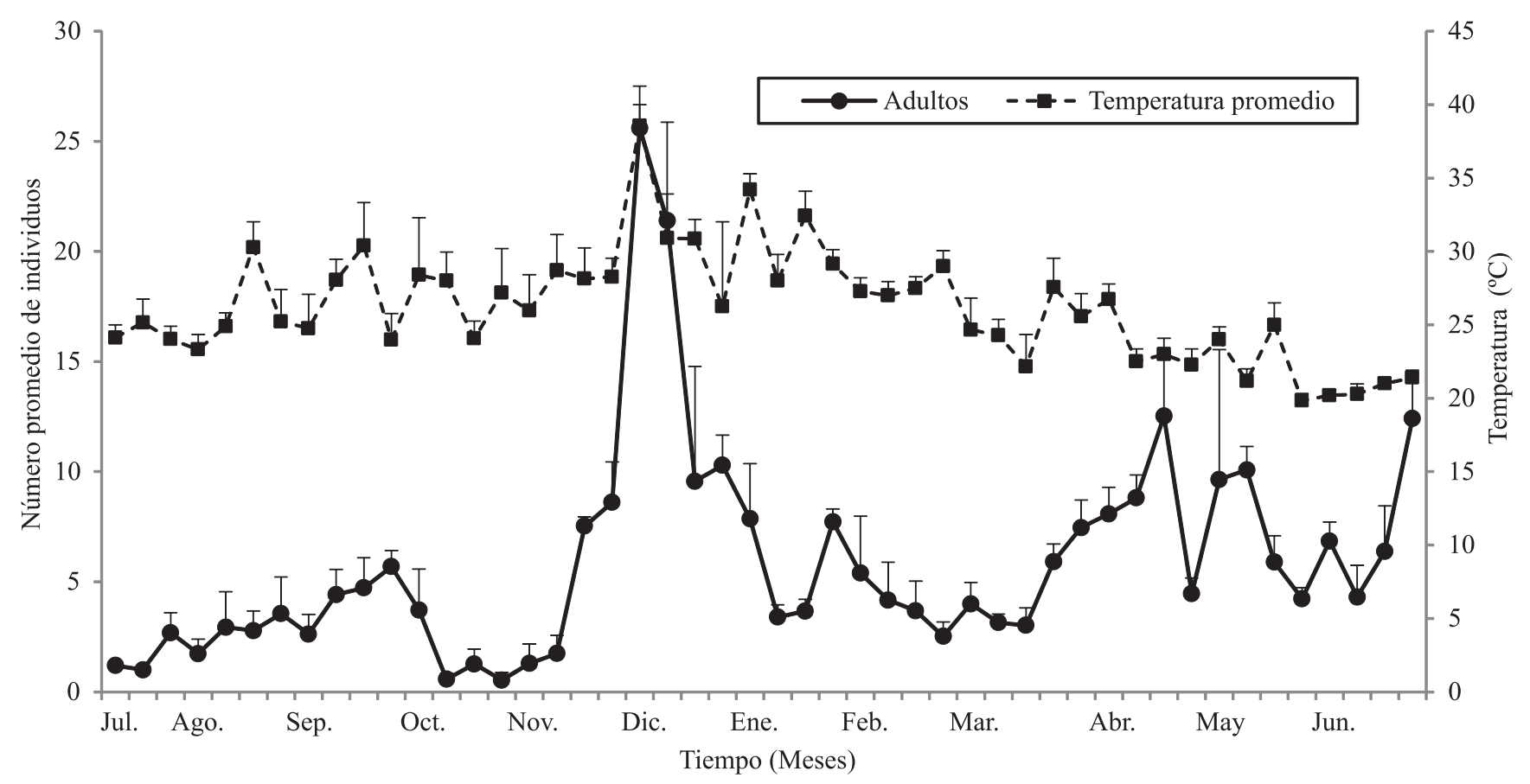

Figura 1. Correlación entre la fluctuación poblacional promedio mensual de adultos de Neohydatothrips signifer en maracuyá, en el municipio de Suaza (2009/2010), con respecto a la temperatura en la misma semana $(\mathrm{p}=0,0032)$. Coeficiente de correlación de Pearson al 5\% de probabilidad. 
el cual empiezan a aparecer las primeras estructuras reproductivas de la planta (Figura 2). Siguiendo con esta tendencia, el máximo pico poblacional se registró en el estado de desarrollo R1, en que se evidenció el incremento de la población con la llegada de la fase reproductiva. Karban \& Agrawal (2002) mencionan que los insectos, para optimizar su alimentación, pueden identificar las plantas que contienen mejor contenido nutricional y, dentro de estas sus órganos vegetales, para alimentarse; las plantas en floración son las más atacadas, por presentar moléculas fáciles de digerir como aminoácidos, los cuales se encuentran mayormente disponibles en las plantas en fase de brotamiento y floración. Además, la alta densidad poblacional del trips, en la etapa de floración, también coincidió con periodos de alta temperatura. Según lo observado en este estudio, se puede inferir que la temperatura es más determinante en el aumento de la densidad poblacional de $N$. signifer, ya que durante el transcurso de la toma de datos se volvieron a presentar periodos de floración, y la densidad poblacional del trips no aumentó de la misma manera como ocurrió cuando hubo aumento la temperatura.
Se presentaron diferencias significativas en cuanto a la incidencia del trips en las estructuras muestreadas, y la mayor incidencia del insecto se registró en los terminales vegetativos con respecto a los botones florales (Cuadro 1). En total, se registraron 16.338 individuos; de estos, 13.576 se encontraron en terminales y 2.762 en botones florales, lo que muestra que también se presentan en mayor número en los terminales vegetativos. Igualmente, se presentaron diferencias significativas en la comparación entre los promedios de la densidad poblacional del trips de acuerdo a su estado de desarrollo de ninfa y adulto con cada estructura, lo que evidenció que tanto adultos como ninfas del insecto también se encuentran en mayor numero en los terminales. Algunos trabajos realizados sobre trips registran que la mayor incidencia de este insecto se observa cuando la planta llega o está en periodo de floración (Ávila Quezada et al., 2002; Urías-López et al., 2007; Kasina et al., 2009). Sin embargo, estos estudios no describen en que parte de la planta el insecto se ubicó después de que hizo su aparición. En el presente estudio, también se encontró que la densidad poblacional de $N$. signifer aumentó cuando la planta llegó a su periodo reproductivo;

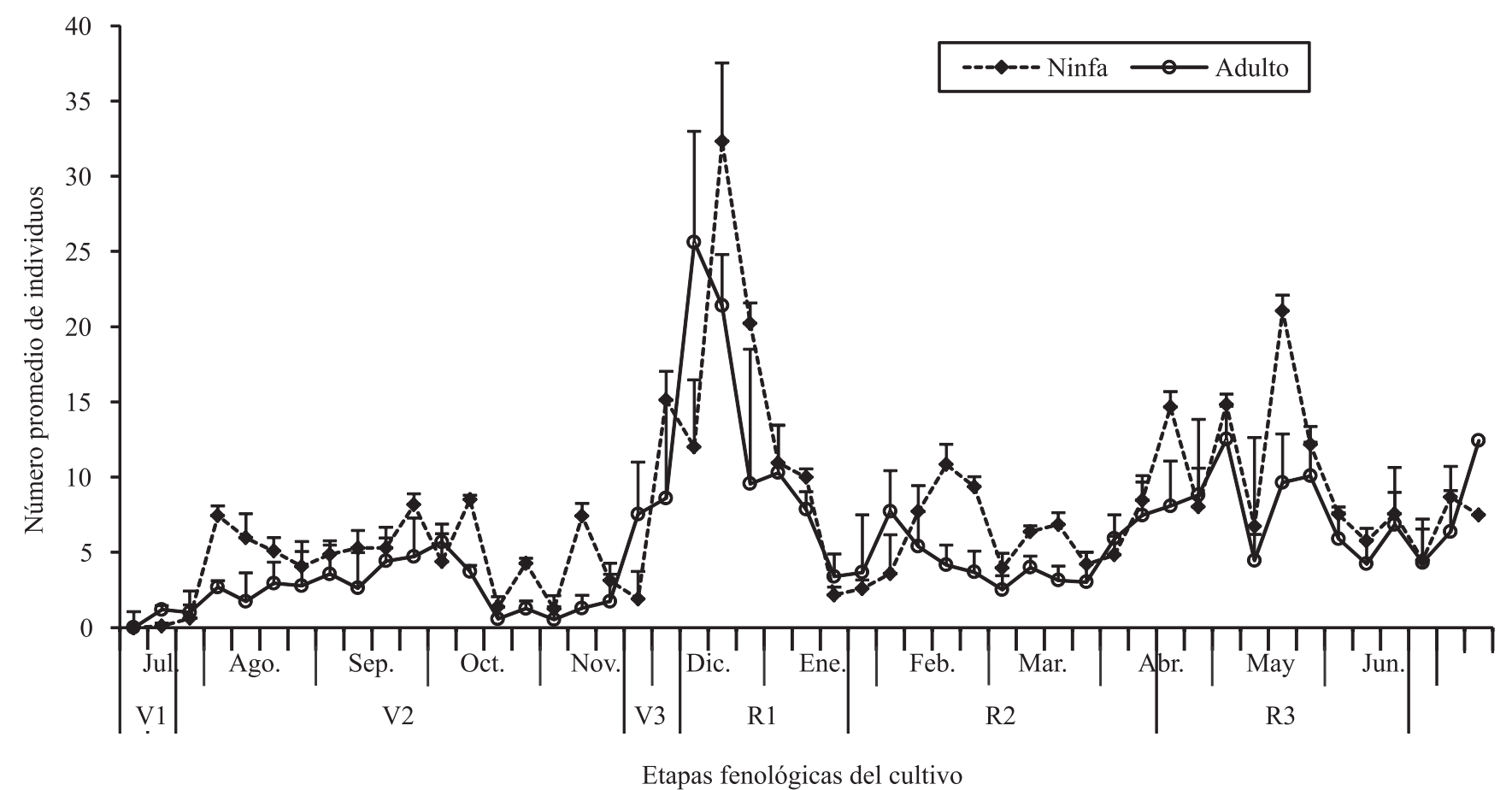

Figura 2. Fluctuación poblacional promedio mensual de ninfas y adultos de Neohydatothrips signifer en maracuyá, con respecto a las etapas de desarrollo del cultivo, en el municipio de Suaza (2009/2010). V1, V2 y V3, etapas vegetativas; R1, R2 y R3, etapas reproductivas. 
sin embargo, esto no significó que la estructura de la planta que presentara la mayor incidencia y densidad poblacional del insecto hubieran sido los botones florales, sino que, para este caso, fueron los terminales vegetativos, lo que coincide con lo expuesto por Jaramillo Vásquez et al. (2009) y Contreras \& Zamar (2010), quienes mencionan que los trips, en el cultivo de maracuyá, se localizan sobre las yemas terminales. Lo anterior sugiere que el muestreo del insecto en el cultivo debe realizarse preferencialmente sobre los terminales vegetativos de la planta.

No se presentaron diferencias significativas en cuanto a la incidencia de ninfas y adultos en los tercios de la planta. En los terminales vegetativos y botones florales, el porcentaje de incidencia de las ninfas osciló entre el 65 y $70 \%$ y el 28 y $31 \%$, respectivamente, y en los adultos osciló entre el 60 y $66 \%$ en los terminales vegetativos y 19 y $23 \%$ en los botones florales. Igualmente, no se presentaron diferencias significativas, cuando se comparó la densidad promedio de las ninfas en los tercios de la rama principal; en los terminales y botones florales, ubicados en cada uno de estos tercios, la densidad osciló entre 2,7 y 3,3 ninfas por terminal y, 0,5 y 0,6 ninfas por botón. Igualmente, no se registraron diferencias significativas para la densidad promedio de adultos en terminales y botones ubicados en cada uno de los tercios, en los que la densidad osciló entre 2,3 y 2,4 adultos por terminal y 0,3 y 0,4 adultos por botón. El hecho de que no se hayan encontrado diferencias

Cuadro 1. Densidad promedio de Neohydatothrips signifer, sobre terminales vegetativos y botones florales, en el cultivo de maracuyá en el municipio de Suaza-Huila, Colombia, 2009/2010.

\begin{tabular}{lccc}
\hline Tratamiento & Total (ninfas + adultos) & Ninfas & Adultos \\
\hline Terminales vegetativos & $4,69 \pm 0,50 \mathrm{a}$ & $2,69 \pm 0,34 \mathrm{a}$ & $1,99 \pm 0,29 \mathrm{a}$ \\
Botones florales & $0,93 \pm 0,04 \mathrm{~b}$ & $0,61 \pm 0,02 \mathrm{~b}$ & $0,32 \pm 0,02 \mathrm{~b}$ \\
\hline
\end{tabular}

Medias \pm error estándar seguidas por letras iguales, en las columnas, no difieren entre si, por el test t de Student, al 5\% de probabilidad. en cuanto a la incidencia y densidad poblacional del trips, entre los tercios de la rama principal, puede deberse a que cada uno de estos presenta condiciones micrometeorológicas similares en la planta. Esto ocurre básicamente porque el tipo de crecimiento de la planta hace que la distancia desde el suelo a cada tercio sea aproximadamente igual para todos, y esto conlleva probablemente a que todos los tercios estén expuestos a similares condiciones ambientales. Así mismo, en todos los tercios se producen tejidos en crecimiento (terminales y botones florales) lo que genera la misma oferta de alimento en cada uno de ellos para el insecto. Esto último concuerda con Morse \& Hoddle (2006), quienes mencionan que el ataque de trips no se reduce a ciertas áreas, sino que se extiende a todas las áreas con tejido en crecimiento de la planta. Este comportamiento del insecto en la planta sugiere que el muestreo del trips puede hacerse tomando cualquier terminal en la planta, debido a que existe la misma probabilidad de encontrar el trips en cualquiera de estos, independientemente de su ubicación.

El patrón de disposición espacial que presentó $N$. signifer para adultos y ninfas, durante los diferentes estados de desarrollo fenológico del cultivo, fue de tipo agregado según la distribución de Poisson con empleo de la prueba de chi-cuadrado, la prueba de la razón $\mathrm{V}^{2} / \mu$ y el índice de agregación $\mathrm{K}$ de la distribución binomial negativa (Cuadro 2). Este tipo de patrón de distribución en trips es probablemente el predominante, ya que ha sido el mayormente registrado en otros cultivos, como en alcornoque (Quercus suber L.) (Soria et al., 2003), aguacate (Persea americana Mill.) (Aranda González, 2004) y uva (Vitis vinifera L.) (Mujica et al., 2007). Existen muchas causas probables para la formación de un patrón agregado, según Pielou (1977), entre ellos están los factores intrínsecos y extrínsecos. Los factores intrínsecos corresponden a las interacciones sociales, tales como la organización para realización de tareas como la búsqueda del alimento o la crianza. En los trips,

Cuadro 2. Distribución espacial de Neohydatothrips signifer, por estado de desarrollo fenológico del cultivo de maracuyá, para adultos y ninfas, en el municipio de Suaza-Huila, Colombia, 2009/2010.

\begin{tabular}{|c|c|c|c|c|c|c|c|c|c|c|c|c|}
\hline \multirow[t]{2}{*}{ Variável } & \multicolumn{2}{|c|}{$\mathrm{V} 1$} & \multicolumn{2}{|c|}{$\mathrm{V} 2$} & \multicolumn{2}{|c|}{$\mathrm{V} 3$} & \multicolumn{2}{|c|}{ R1 } & \multicolumn{2}{|c|}{$\mathrm{R} 2$} & \multicolumn{2}{|c|}{ R3 } \\
\hline & $\mathrm{TA}$ & $\mathrm{TI}$ & $\mathrm{TA}$ & $\mathrm{TI}$ & TA & $\mathrm{TI}$ & TA & $\mathrm{TI}$ & TA & $\mathrm{TI}$ & TA & $\mathrm{TI}$ \\
\hline $\mathrm{X}^{2}$ para Poisson ${ }^{(1)}$ & $\leq 0,001$ & $\leq 0,001$ & $\leq 0,001$ & $\leq 0,001$ & $\leq 0,001$ & $\leq 0,001$ & $\leq 0,001$ & $\leq 0,001$ & $\leq 0,001$ & $\leq 0,001$ & $\leq 0,001$ & $\leq 0,001$ \\
\hline $\mathrm{V}^{2} / \mu$ & 1,66 & 2,86 & 5,45 & 11,54 & 2,86 & 4,84 & 4,92 & 5,95 & 2,72 & 5,12 & 3,00 & 3,16 \\
\hline Binomial negativa $(\mathrm{K})$ & 1,15 & 0,33 & 0,59 & 0,55 & 0,88 & 0,34 & 1,04 & 0,92 & 0,88 & 0,65 & 1,26 & 1,57 \\
\hline
\end{tabular}

${ }^{(1)} \mathrm{X}^{2}$ significa que no se ajusta a la distribución de Poisson a 1\% de probabilidadee indica que el tipo de distribución es agregado. TA, trips adulto; TI, trips inmaduro. 
aunque este comportamiento social no es el predominante (Ananthakrishnan, 2008), ya ha sido registrado en algunas especies como Elaphrothrips tuberculatus (Hood, 1908) (Thysanoptera: Phlaeothripidae), Actinothrips trichaetus (Hood, 1935) (Thysanoptera: Phlaeothripidae) y Anactinothrips gustaviae (Mound \& Palmer, 1983) (Thysanoptera: Phlaeothripidae); en esta última especie, se encontró una "colonia" que se movía en bloque en búsqueda del alimento. Entre los factores extrínsecos se mencionan comportamientos defensivos y aprovechamiento de parches de alta calidad con el despoblamiento de zonas pobres (Pielou, 1977). De acuerdo a este resultado, el muestreo para $N$. signifer, durante todas las etapas de desarrollo fenológico del cultivo, debe hacerse con la distribución lo mejor posible del número de muestras, y cubrir el cultivo en su totalidad para así aumentar su eficiencia, ya que el patrón de distribución espacial que presenta el insecto es de tipo agregado, lo que significa que los individuos se agrupan en aglomerados o parches, dejando porciones del espacio relativamente desocupadas, lo cual disminuye la probabilidad de encontrarlo en el cultivo cuando se realizan los muestreos.

La distribución que correspondió a $N$. signifer en el cultivo de maracuyá fue de tipo agregada, por tanto el modelo matemático a utilizar para el cálculo del número de muestras es el binomial negativo, cuya fórmula, sugerida por Rojas (1964), fue descrita en la metodología. El número de muestras se calculó para el estado de desarrollo denominado formación del fruto (R2), con el índice de agregación (K) obtenido de la distribución de la binomial negativa y la media poblacional registrada en los muestreos para ese estado de desarrollo de la planta. Se escogió esta etapa fenológica, por ser la más importante en el cultivo desde el punto de vista agronómico $y$, además, porque es la más representativa, ya que después de la primera floración, el cultivo presentara flores, botones florales y frutos por el resto de su vida útil. El cálculo sugiere que se deben muestrear como mínimo 22 terminales por hectárea (Cuadro 3). Este tamaño de muestra no

Cuadro 3. Cálculo del número de muestras de Neohydatothrips signifer por hectárea, en el cultivo de maracuyá.

\begin{tabular}{lccc}
\hline № de muestras por & \multicolumn{3}{c}{ Componentes del estimador } \\
\cline { 2 - 4 } hectárea $(\mathrm{n})$ & $\mathrm{K}$ & $\mathrm{CV}$ & $\mu$ \\
\hline $22,49 \simeq 22$ & 0,65 & 0,3 & 2,058 \\
\hline
\end{tabular}

$\mathrm{K}$, Índice de dispersión; CV, Coeficiente de variación; $\mu$, Promedio poblacional del insecto en los muestreos. es alto, teniendo en cuenta que el insecto presentó un tipo de distribución agregado, lo que conlleva a mayores unidades requeridas para el muestreo. Así mismo, el esfuerzo de muestreo de 22 terminales por hectárea no parece desgastante para el agricultor, pues es abundante el número de terminales vegetativos que produce la planta y la facilidad para encontrarlos durante cualquier recorrido que se haga dentro del lote. Finalmente, es importante considerar que los datos utilizados en todo el estudio corresponden a una temporada, una localidad y sólo una variedad frutal; por lo tanto, no se puede generalizar los resultados obtenidos, a menos que se repita el estudio durante algunas temporadas y en distintas zonas, para poder así construir modelos de agregación que sean representativos en la mayoría de las situaciones posibles.

\section{Conclusiones}

1. En épocas con temperaturas altas y de periodos de floración de la planta, la población de trips tienden a incrementarse extraordinariamente, por eso, se debe aumentar la rigurosidad y el número de los muestreos.

2. El muestreo para Neohydatothrips signifer en el cultivo debe hacerse con un mínimo de 22 terminales vegetativos, distribuyendo lo mejor posible las unidades a muestrear dentro del lote.

3. El muestreo para trips en el cultivo de maracuyá debe hacerse sobre los terminales vegetativos de la planta, sin importar la posición del terminal en ella, ya que existe la misma probabilidad de encontrar los trips en cualquiera de estos, independientemente de su ubicación.

\section{Agradecimientos}

Al Ministerio de Agricultura y Desarrollo Rural de Colombia, por el apoyo financiero; al agricultor Genaro Cuellar, por disponibilizar su finca para el desarrollo de esta investigación.

\section{Referencias}

ANANTHAKRISHNAN, T.N. The social biology and adaptability in mycophagous tubuliferan thrips. Current Science, v.6, p.708-709, 2008.

ARANDA GONZÁLEZ, P.A. Trips del palto (Heliothrips haemorrhoidalis Bouche): disposición espacial a nivel de huerto 
y determinación del número de muestras a utilizar en paltos. 2004. 67p. Taller (Licenciatura) - Pontificia Universidad Católica de Valparaiso, Quilota.

ARTURO GUERRERO, J. Estudio de residuos de plaguicidas en frutas y hortalizas en áreas específicas de Colombia. Agronomía Colombiana, v.3, p.198-209, 2003.

ÁVILA QUEZADA, G.D.; TELÍZ ORTIZ, D.; GONZÁLEZ HERNÁNDEZ, H.; VAQUERA HUERTA, H.; TIJERINA CHÁVEZ, L.; JOHANSEN NAIME, R.; MOJICA GUZMÁN, A. Dinámica espacio-temporal de la roña (Elsinoe perseae), el daño asociado a trips y antracnosis (Glomerella cingulata) del aguacate en Michoacán, México. Revista Mexicana de Fitopatología, v.20, p.77-87, 2002.

BUENO, J.M.; CARDONA, C. Umbral de acción para Thrips palmi (Thysanoptera: Thripidae) en habichuela en el Valle del Cauca, Colombia. Revista Colombiana de Entomología, v.29, p.51-55, 2003.

CHEN, X.; YUAN, L.; DU, Y.; ZHANG, Y.; WANG, J. Cross-resistance and biochemical mechanisms of abamectin resistance in the western flower thrips, Frankliniella occidentalis. Pesticide Biochemistry and Physiology, v.101, p.34-38, 2011.

CONTRERAS, E.F.; ZAMAR, M.I. Morfología de los estados inmaduros y adulto de Neohydatothrips denigratus (De Santis) (Thysanoptera: Thripidae), con datos sobre su biología. Neotropical Entomology, v.39, p.384-390, 2010.

GARCÍA LOZANO, J.; FIGUEROA, L.; OCAMPO, L.A.; FORERO LONGAS, F.; VERA, L.F.; SEGURA, J.D.; GÓMEZ, B.A. Generación de un modelo de zonificación edafoclimática y socioeconómica a nivel departamental y municipal, para la producción de mora, lulo, maracuyá, chulupa, granadilla, uva y tomate de árbol en el departamento del Huila. Vía Mosquera: Corporación Colombiana de Investigación Agropecuaria, 2007. 96p. (Informe final proyecto. Convenio especial de cooperación técnica y científica, 491/2005).

GONZÁLEZ-ZAMORA, J.E.; GARCIA-MARÍ, F. The efficiency of several sampling methods for Frankliniella occidentalis (Thysan., Thripidae) in strawberry flowers. Journal of Applied Entomology, v.127, p.516-521, 2003.

JARAMILLO VÁSQUEZ, J.; CARDENAS ROCHA, J.; OROZCO ÁVILA, J. Manual sobre el cultivo de maracuyá (Passiflora edulis) en Colombia. Vía Mosquera: Corporación Colombiana de Investigación Agropecuaria, 2009. 80p.

KARBAN, R.; AGRAWAL, A. Herbivore offense. Annual Review of Ecology and Systematics, v.33, p.641-664, 2002.

KASINA, M.; NDERITU, J.; NYAMASYO, G.; WATURU, C.; OLUBAYO, F.; OBUDHO, E.; YOBERA, D. Within-plant distribution and population dynamics offlower thrips (Thysanoptera: Thripidae) infesting french beans (Phaseolus vulgaris L.) in Kenya. Spanish Journal of Agricultural Research, v.3, p.652-659, 2009.

KOVANCI, O.B.; KOVANCI, B.; NIMET, S.; GENCER, N.S. Sampling and development of economic injury levels for Anthonomus rubi Herbst adults. Crop Protection, v.24 p.1035-1041, 2005.
MADADI, H.; DASHTI, F.; ENKEGAARD, A. Spatial distribution of Thrips tabaci and development of a fixed-precision sampling plan for greenhouse cucumber. Journal of Entomology, v.8, p.280-287, 2011.

MARCO, V. Modelización de la tasa de desarrollo de insectos en función de la temperatura. Aplicación al manejo integrado de plagas mediante el método de grados-día. Boletín Sociedad Entomológica Aragonesa, n.28, p.147-150, 2001.

MICHELA, J.F.; JUÁREZ, M.; FIORENTINO, D.C.; NOTARIO, A.; CASTRESANA, L. Distribución espacial y su variabilidad con respecto al tiempo de una población de Micrapate wagneri Lesne (Coleoptera: Bostrichidae) en un rodal de Prosopis nigra (Gris) Hieron., en Santiago del Estero, Argentina. Boletín de Sanidad Vegetal. Plagas, v.26, p.11-14, 2000.

MORSE, J.G.; HODDLE, S.M. Invasion biology of thrips. Annual Review of Entomology, v.51, p.67-89, 2006.

MOUND, L.A.; PALMER, J.M. Spore-feeding Thysanoptera of the genus Anactinothrips with a new sub-social species from Panama. Journal of Natural History, v.5, p.789-797, 1983.

MUJICA, M.V.; SCATONI, I.B.; FRANCO, J.; NUÑEZ, S.; BETANCOURT, C.M. Fluctuación poblacional de Frankliniella occidentalis (Pergande) (Thysanoptera: Thripidae) en "Vitis vinifera" L. cv. Italia en la zona Sur de Uruguay. Boletín de Sanidad Vegetal. Plagas, v.4, p.457-468, 2007.

NAULT, B.A.; SHELTON, A.M. Impact of insecticide efficacy on developing action thresholds for pest management: a case study of onion thrips (Thysanoptera: Thripidae) on onion. Journal of Economic Entomology, v.103, p.1315-1326, 2010.

PARK, C.-G.; KIM, Y.-H.; LEE, J.-H. Parameter estimation for a temperature-dependent development model of Thrips palmi Karny (Thysanoptera: Thripidae). Journal of Asia-Pacific Entomology, v.13, p.145-149, 2010.

PÉREZ, D.; IANNACONE, J. Fluctuación y distribución espacio-temporal de Tuthillia cognata (Hemiptera: Psyllidae) y de Ocyptamus persimilis (Diptera, Syrphidae) en el cultivo de camu-camu Myrciaria dubia (Myrtaceae) en Ucayali, Perú. Revista Brasileira de Entomologia, v.53, p.635-642, 2009.

PIELOU, E.C. Mathematical ecology. New York: John Wiley \& Sons, 1977. 385p.

RAMÍREZ-DÁVILA, J.; GONZÁLEZ-ANDÚJAR, J.L.; OCETE, R.; LÓPEZ MARTÍNEZ, M.A. Descripción geoestadística de la distribución espacial de los huevos del mosquito verde Jacobiasca lybica (Bergenin \& Zanon) (Homoptera: Cicadellidae) en viñedo: modelización y mapeo. Boletín de Sanidad Vegetal. Plagas, v.28, p.87-95, 2002.

RIVERA, B.; MIRANDA, D.; ÁVILA, L.A,; NIETO, A.M. Manejo integral del cultivo de la granadilla (Passiflora ligularis Juss.). Manizales: Litoas, 2002. 130p.

ROJAS, B.A. La binomial negativa y la estimación de intensidad de plagas en el suelo. Fitotecnia Latinoamericana, v.1, p.27-36, 1964.

SALAMANCA BASTIDAS, J.; VARÓN DEVIA, E.H.; SANTOS AMAYA, O. Cría y evaluación de la capacidad de depredación de Chrysoperla externa sobre Neohydatothrips signifer, trips 
plaga del cultivo de maracuyá. Corpoica Ciencia y Tecnología Agropecuaria, v.11, p.31-40, 2010.

SANTOS A., O.; VARÓN D., E.H.; GAIGL, A.; FLORIANO, J.A. Nivel de daño económico para Neohydatothrips signifer (Thysanoptera: Thripidae) en maracuyá en el Huila, Colombia. Revista Colombiana de Entomología, v.38, p.23-29, 2012.

SORIA, F.J.; VILLAGRÁN, M.; JIMÉNEZ, A.; OCETE, M.E. Distribución temporal y espacial de Oxythrips quercicola Bagnall (Thysanoptera: Thripidae) en amentos masculinos de alcornoque. Boletín de Sanidad Vegetal. Plagas, v.29, p.505-509, 2003.

TRIPLEHORN, C.A.; JOHNSON, N.F. Estudo dos insetos. São Paulo: Cengage Learning, 2011. 808p.

URÍAS-LÓPEZ, M.A.; SALAZAR-GARCÍA, S.; JOHANSEN-NAIME, R. Identificación y fluctuación poblacional de especies de trips (Thysanoptera) en aguacate "Hass" en Nayarit, México. Revista Chapingo. Serie Horticultura, v.13, p.49-54, 2007.

Recibido en 19 de abril de 2012 y aprobado en 13 de outubro de 2012 